\title{
The use of the laser confocal scanning microscopy to measure resin remnants on customized lingual bracket
}

\author{
Can Kuskonmaz ${ }^{1}$, Alberto De Stefani ${ }^{1}$, Gilberto Artioli ${ }^{2}$, Matteo Zanarini ${ }^{3}$, Giulio Alessandri Bonetti ${ }^{3}$, \\ Giovanni Bruno ${ }^{1 *}$ (D) and Antonio Gracco ${ }^{1}$
}

\begin{abstract}
Background: The study aimed to evaluate the permanence of resin and enamel remains on lingual brackets at the end of orthodontic treatment and after the debonding procedure. The evaluation of resin remnants on customized lingual brackets bases has never done before in other studies because they are curved, and traditional techniques are not applicable.

Methods: The sample consisted of 100 lingual brackets (25 incisors, 25 canines, 25 premolars, 25 molars) scanned with a confocal laser microscope (OLS4000). We measured the brackets' surface and the area of resin remnants with the software of the microscope. Median and quartiles were presented to describe the data. ARI calculation was indirect for each tooth, measuring the resin remnants to the total surface of the bracket. The Kruskal-Wallis test and Fisher test were applied respectively to compare the percentages of remnants and the frequencies of the ARI between the four groups.

Results: After the analyses, 13 brackets had no adhesive remnants (ARI 0), 29 brackets had less than 50\% of resin remnants (ARI 1), 50 brackets had more than 50\% of resin remnants (ARI 2), and 8 brackets had 100\% of adhesive (ARI 3). Canines brackets presented the lower amount of resin followed by premolars, incisors, and molars.

Conclusion: Lingual brackets showed a high frequency of ARI $=2$. The median percentage of the bracket surface covered by resin was $41 \%$. We observed a slight tendency of more resin remnants on molar brackets, due to halfpad configuration. The authors suggest paying attention during the debonding procedure of molar brackets since a stronger connection between the adhesive and the bracket mesh means a higher risk of enamel damage.
\end{abstract}

Keywords: Lingual orthodontics, debonding, ARI, confocal microscope

\section{Background}

At the end of the orthodontic treatment with labial or lingual appliances, orthodontists have to remove brackets, residual composite, and adhesive remnants from the teeth surfaces, trying to restore the pretreatment condition without damaging the enamel surface

\footnotetext{
* Correspondence: giobruno93@gmail.com

'Faculty of Dentistry, University of Padova, via Giustiniani 2 -, 35100 Padova, Italy

Full list of author information is available at the end of the article
}

[1-3]. There are different factors associated with this procedure, for example, the kind of bonding protocols and materials but also the tools for bracket removal and burs sequence $[4,5]$.

In medical literature, there are not universally approved protocols for the clean-up of adhesive resin after bracket removal, and no instrument can achieve complete resin removal without scratching or grooving the enamel surface. Damage to the enamel surface during debonding procedure has been reported in vitro [6-

(c) The Author(s). 2020 Open Access This article is licensed under a Creative Commons Attribution 4.0 International License, which permits use, sharing, adaptation, distribution and reproduction in any medium or format, as long as you give appropriate credit to the original author(s) and the source, provide a link to the Creative Commons licence, and indicate if changes were made. The images or other third party material in this article are included in the article's Creative Commons licence, unless indicated otherwise in a credit line to the material. If material is not included in the article's Creative Commons licence and your intended use is not permitted by statutory regulation or exceeds the permitted use, you will need to obtain permission directly from the copyright holder. To view a copy of this licence, visit http://creativecommons.org/licenses/by/4.0/ The Creative Commons Public Domain Dedication waiver (http://creativecommons.org/publicdomain/zero/1.0/) applies to the data made available in this article, unless otherwise stated in a credit line to the data. 
$8]$ and in vivo studies $[9,10]$. Bond failure during the bracket removal procedure can occur in different modalities: adhesive failure is when it occurs at the adhesivebracket interface or the adhesive-enamel interface, cohesive failure is when it occurs within the adhesive. The most frequent scenario is generally a combination is a mixed failure when a combination of adhesive and cohesive failure happens [11]. Different studies evaluated the damage of the dental surface during debonding, demonstrating that a higher risk occurs with an adhesive failure between the resin and the enamel [11-13]. This event can occur with metal brackets but more often with ceramic ones [14].

At the date, only a few studies evaluated the debonding forces, and shear bond strength and bracket base remnants of customized lingual appliance [15]. The principal limitations of these studies are the in-vitro protocols and that authors generally perform the tests only on premolars brackets. The customized lingual bracket bases are often very curved, and they do not permit to measure the adhesive remnant index (ARI) [16], with traditional procedures such as digital photography or scanning electron microscope (SEM) analysis. Confocal scanning microscope presents the advantage of capturing 3D images and found applications in different fields $[17,18]$ but never in orthodontic for brackets evaluation. The scanning procedure, however, requires a long period of training and the acquisition for every single bracket last between 60 and 90 minutes.

This research aims to perform the first in-vivo analysis of the composite remnants on the bracket surface of a customized lingual appliance.

\section{Methods}

The authors of the present study collected one hundred lingual brackets ( 25 incisors, 25 canines, 25 premolars, and 25 molars) at the end of orthodontic treatment with customized lingual appliance (Incognito ${ }^{\mathrm{m}}, 3 \mathrm{M}$ ) from 25 patients. Every patient was collected randomly one bracket per type with a general 50/50 ratio between the maxillary and the mandibular dental arches, according to the following inclusion criteria: no antecedent orthodontic treatment, no history of dental trauma, uninjured lingual surface, and no bleaching in the previous 12 months. Patients presenting a Bolton index discrepancy were excluded as well as patients that required an extraction treatment, orthognathic surgery or had impacted teeth. Furthermore, we excluded treatments that lasted more than 24 months. Regarding the molars and premolars, brackets presenting an excessive curvature and $90^{\circ}$ undercuts were excluded from the sample because they will have affected the scanning procedure. Sample size calculation revealed that a minimum of 80 brackets (20 per group) were necessary to identify, with a power of 0.80 and a type I error of $5 \%$, a significant difference in resin remnants percentage between the four groups.

The bonding procedures followed the indirect bonding protocol with the clear silicon tray, provided by the manufacturer instruction, and it involved sandblasting the teeth surface (aluminum oxide 50 micron), etching for 30 seconds with orthophosphoric acid 37\%, universal bonding and Relyx Unicem. The first debonding phase consisted in removing the appliance with an appropriate lingual debonding plier. Secondly, the operator carefully cleaned up the teeth with a debonding carbide burs at slow speed and performed a final step of enamel polishing with finishing points. The same operator performed all bonding and debonding procedures.

Considering the curved surface of lingual brackets, an optical microscope with 20x magnification would have obtained pictures out of focus in some part of the bracket affecting a correct ARI calculation (Fig. 1). This kind of microscope is sufficiently precise for standard vestibular or lingual brackets without a customized base. For this reason, we decided to use a confocal laser microscope (Olympus Lext OLS4000). The confocal laser microscope finds its application for nanometric scale imaging (magnification ranges from 100x to 17000x) and roughness measurements. The highresolution 3D images of the brackets let us calculate the total base and resin remnants surfaces (Fig. 1).

One operator, skilled in confocal laser microscope imaging, acquired all the 3D images. When the total surface of the bracket exceeded the maximum acquiring area the integrated software automatically merges two or more acquisitions. After the scanning phase, the brackets' surface and the area of resin remnants were measured with the software of the microscope by a first operator. A second round of measurements was performed by the same operator and by a second operator on all the brackets to calculate the intraobserver and inter-observer agreement on the ARI.

The values were calculated as the percentage between the total area and the resin surface as an index of the quantity of adhesive on enamel after debonding (Fig. 2).

ARI calculation was indirect for each tooth, measuring the resin remnants to the total surface of the bracket. ARI's categories are considered in according with Sfondrini et al. [19]

- ARI 0 no resin remnants on the bracket surface

- ARI 1 less than a half of the bracket covered by resin remnants

- ARI 2 more than a half of the bracket covered by resin remnants

- ARI 3 all the bracket covered by resin remnants.

Furthermore, the percentage was calculated for each bracket group (Figs. 3 and 4). 


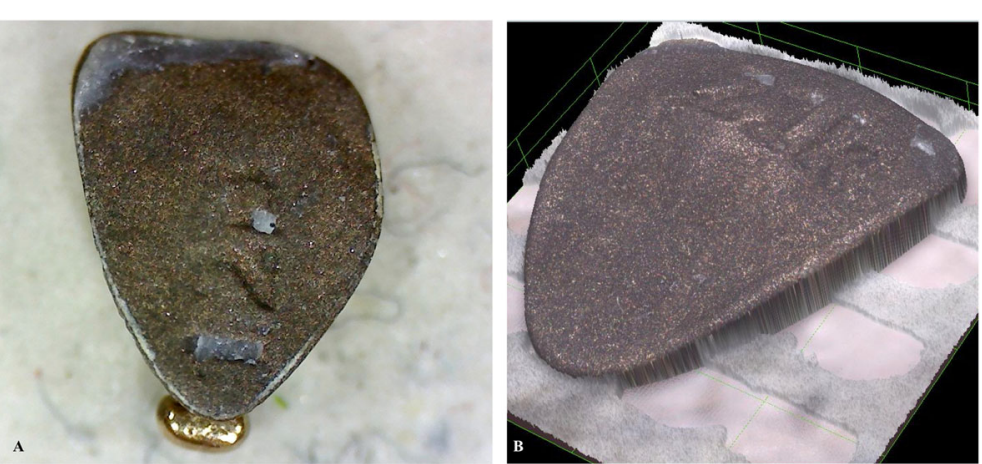

Fig. 1 a Bracket evaluation at the optical microscope 20x; b Three-dimensional scanning with the confocal microscope

\section{Statistical analysis}

Concerning the ARI classification, the inter-observer agreement was assessed with the Fleiss' Kappa and the intra-observer agreement with the Cohen's Kappa. The Shapiro-Wilk test was used to test the normality of the distribution of the primary outcome (resin remnants percentage) evidencing a significance deviation from normality. Median and quartiles were presented to describe the data. The Kruskal-Wallis test and the Fisher test were applied respectively to compare the percentages of remnants and the frequencies of the ARI between the four groups. Differences were considered significant when they reached a p-value $<0.05$. The data analysis was carried out with the aid of R 3.5 (R Foundation for Statistical Computing, Vienna, Austria).

\section{Results}

The sample includes 100 brackets, divided into 25 incisors (13 upper and 12 lower), 25 canines (12 upper and 13 lower), 25 premolars (13 upper and 12 lower), and 25 molars (12 upper and 13 lower). Mean bracket surface in the four groups is reported in Table 1.

To record and measure the amount of adhesive remaining on tooth and bracket surfaces, the adhesive

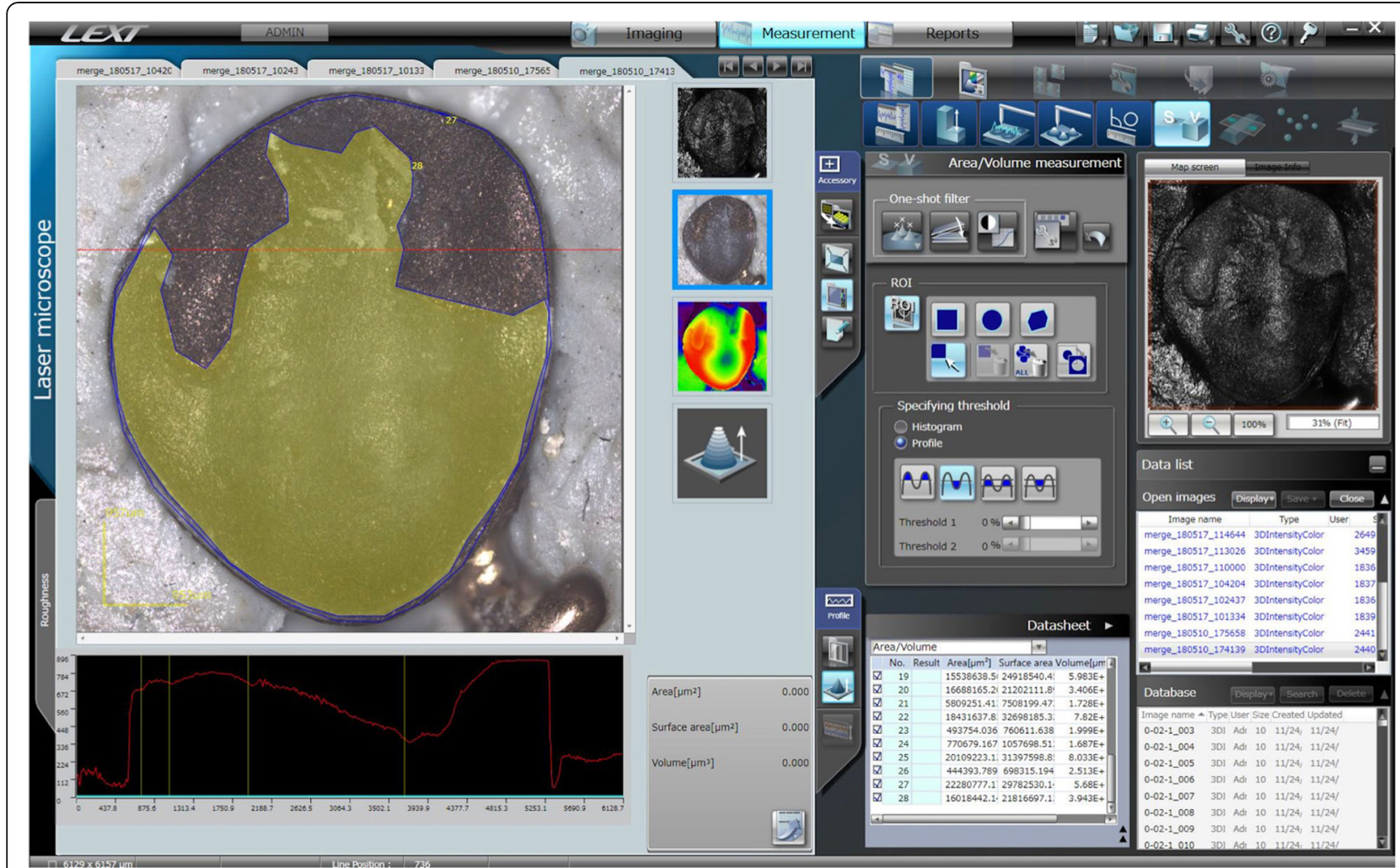

Fig. 2 Brackets' surface and the area of resin remnants were measured with the software of the microscope 

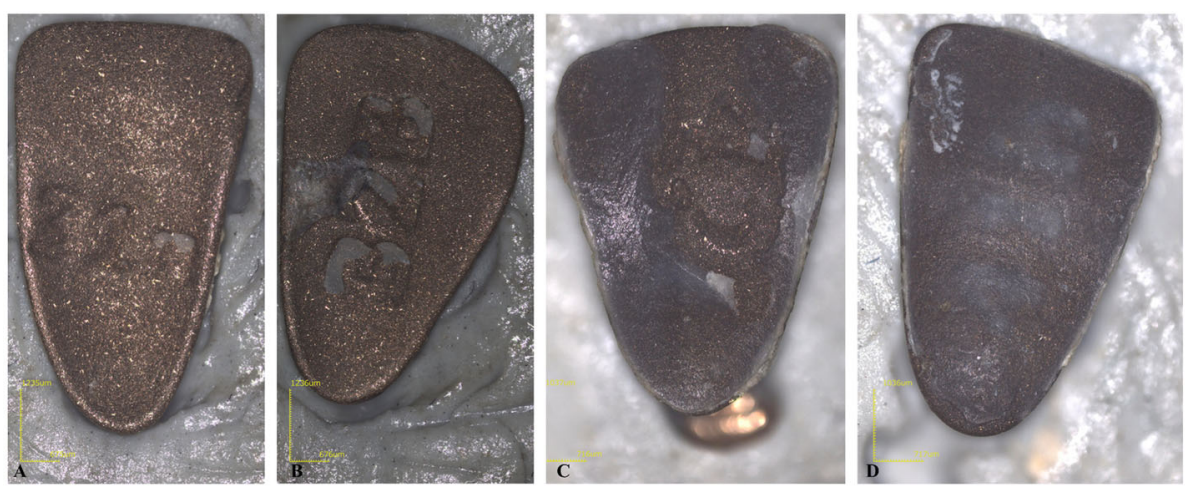

Fig. 3 Incisors: a ARI 3; b ARI 2 c ARI 1 d ARI 0

remnant index (ARI) score was used. This scale allows the allocation of a score $(0,1,2$, or 3$)$ depending on the amount of adhesive detected on the enamel surface.

Excellent inter-observer (two observers: Fleiss' Kappa 0.93 ) and intra-observer (two classifications of ARI index after 30 days: Cohen's Kappa 0.97) matches were observed.

After all the brackets evaluation, the incisors presented 4 brackets with ARI 0,8 were ARI 1,12 were ARI 2 and 1 bracket was ARI 3. The canines presented 1 bracket with ARI 0,5 brackets were ARI 1,18 were ARI 2 and 1 was ARI 3. The premolars presented 4 brackets with ARI 0,7 were ARI 1,9 were ARI 2 and 5 were ARI 3 . Finally, molars presented 4 brackets with ARI 0,9 were ARI 1,11 were ARI 2 and 1 were ARI 3 . Table 1 and in Figs. 5 and 6 extensively report the results in terms of median percentage, quartiles, and ARI distribution in the four groups.

Concerning the whole sample, 13 brackets resulted with no resin surface meaning no adhesive on the bracket, 29 brackets resulted in less than half of adhesive on the surface, 50 brackets resulted in more than half of adhesive on the surface and 8 brackets resulted in all the surface covered of adhesive. Canines are the brackets with the lower remnants of resin followed by premolars, incisors, and molars. Concerning the percentage of resin remnants, Kruskal-Wallis test did not result statistically significant $(p=0.27399)$. No significant differences emerged in the ARI distribution between the four groups, evaluated with the Fisher test $(p=0.1679)$.

\section{Discussion}

Lingual orthodontics, among both young and adult patients, increased in popularity during the last years. Recent studies demonstrated that lingual orthodontics could provide treatment outcomes similar to those achieved with labial appliances.

The orthodontic literature shows many articles that describe the failure of the brackets bonding, the major part of which were made in vitro and on small teeth sample size, usually premolars $[2,5,6,11,13,20]$. There are different limitations of in-vitro studies: the increasing load applied is not representative of the clinical debonding situation that presents a combination of cut, traction, and torsion forces. Furthermore, oral conditions during an orthodontic treatment such as temperature, humidity, salivary $\mathrm{pH}$, and plaque surely influence the debonding result and cannot be simulated in a laboratory.

There are very few studies on the failure of the customized lingual bracket bonding [21-23], for the difficulty of evaluating a curve surface. There are no studies that evaluate it on premolars and molars after orthodontic treatment.
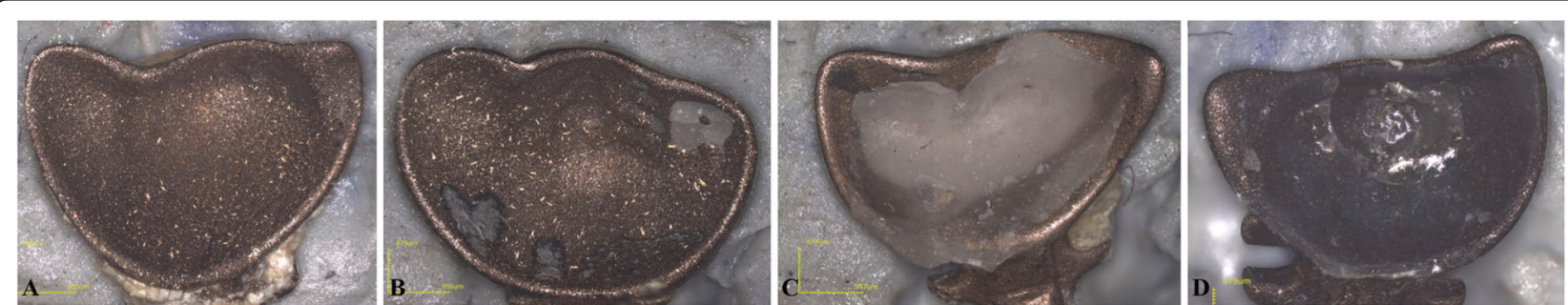

Fig. 4 Premolars: a ARI 3; b ARI 2 c ARI 1 d ARI 0 
Table 1 Resin remnants as percentage for the different teeth

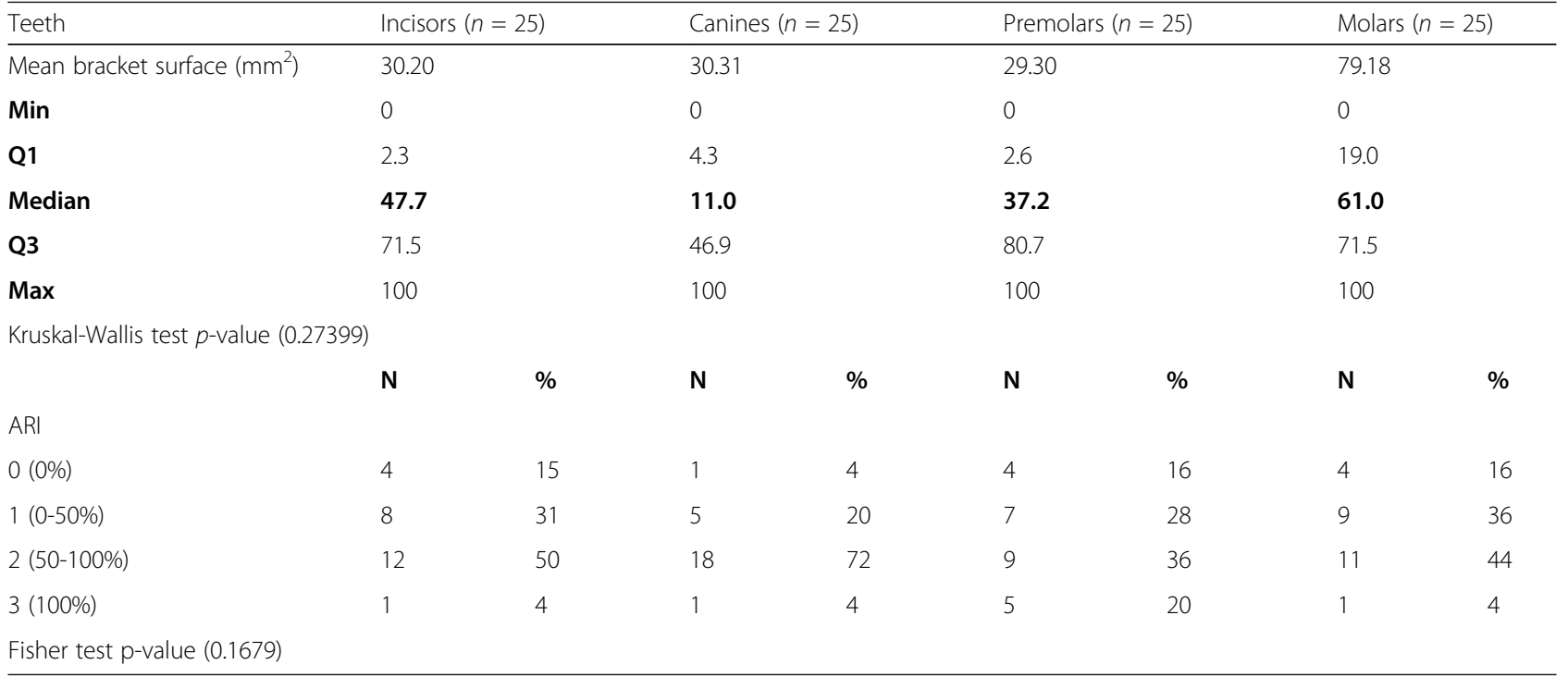

The debonding procedure should preserve the enamel integrity. Resin remnants left on the teeth increase the risk of damaging the enamel during the cleanup procedure with burs. On the other side, its presence on the surface of the brackets is not an advantageous event for the high probability of enamel damage. The frequent prevalence of ARI 2 suggests that the debonding of lingual brackets is comparable with conventional vestibular bracket. Molar customized brackets presented an increased but not statistically significant presence of resin remnant, probably due to the larger bracket surface and their increased convexity. For this reason, the authors of this study suggest paying attention during the debonding procedure of molar brackets since a stronger connection between the adhesive and the bracket mesh means a higher risk of enamel damage [24].

No previous studies evaluated the bracket's base with a confocal laser microscope. The authors captured highresolution 3D images to measure the entire base and resin remnants in a curved surface and considered this method consistent, precise, and reproducible. On the other side, the confocal laser microscope presented some

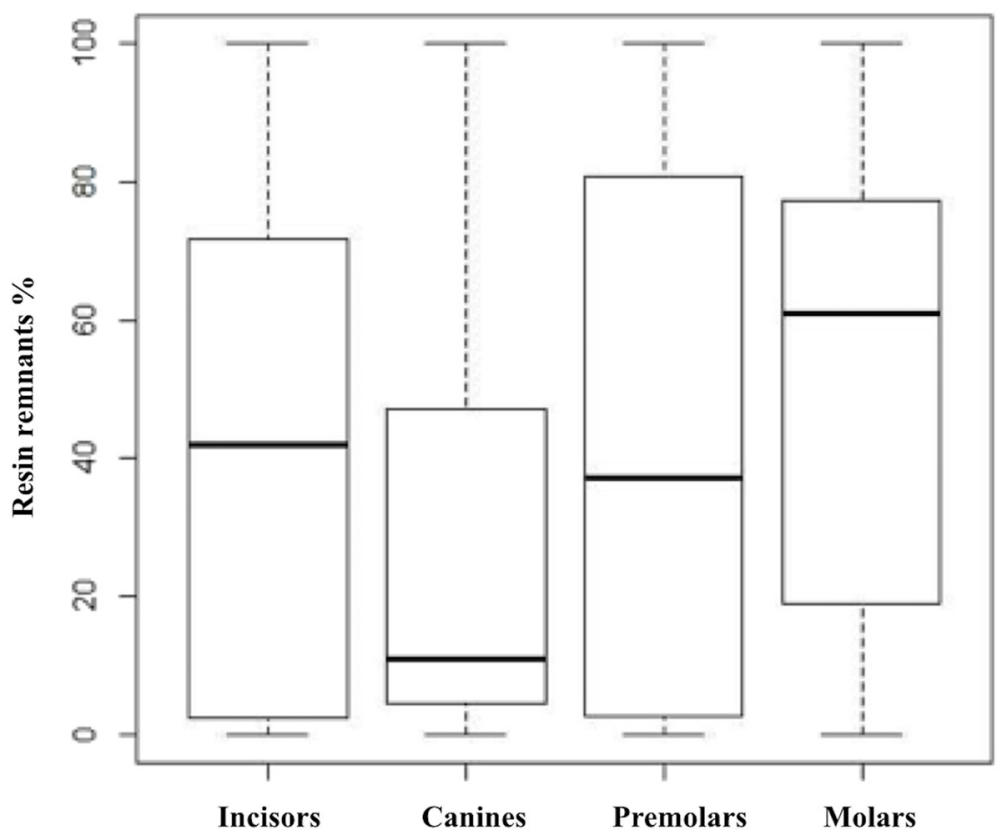

Fig. 5. Resin remnants on the brackets (percentage): boxplot 


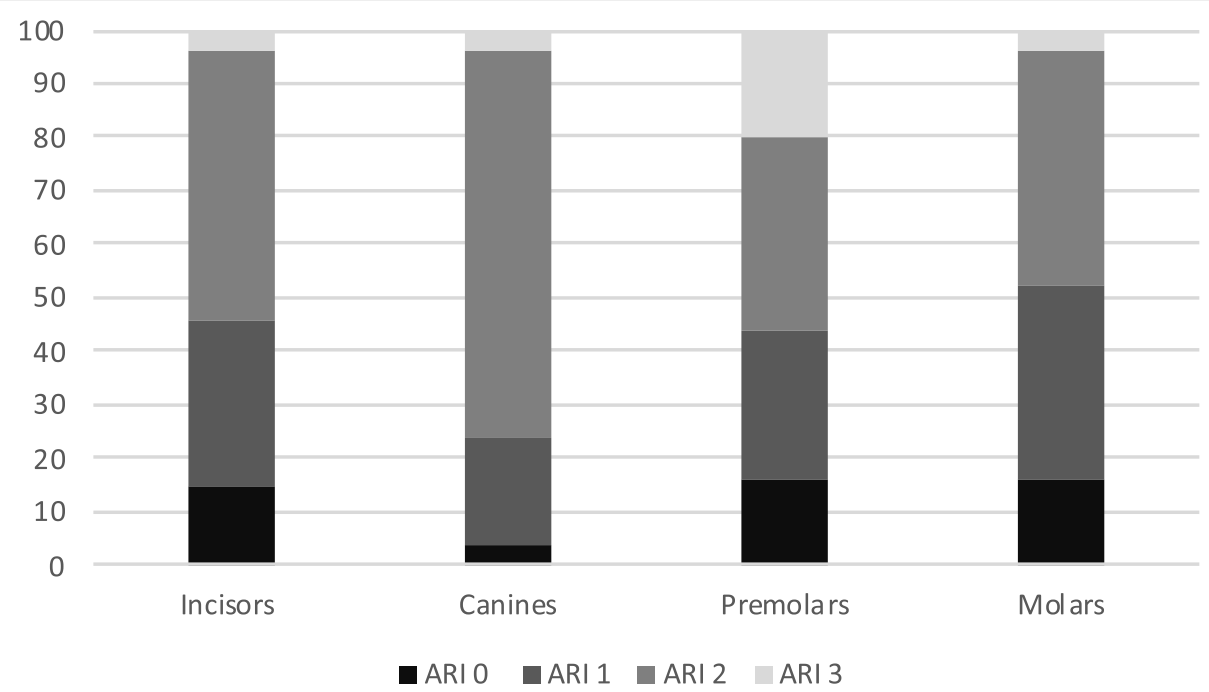

Fig. 6. Resin remnants (ARI): histogram

limits with the brackets with a curvature higher than $85^{\circ}$. Some customized lingual premolars and molars show occlusal extensions with excessive curvature, and for this reason, these brackets were excluded in this study. This study also presents some limitations. First of all, the acquisition is an expensive procedure in cost and time and surely requires a skilled operator. Secondly, the authors did not consider in the study design the percentage of material that can be lost during the debonding procedure.

\section{Conclusion}

No previous studies evaluated ARI on customized lingual brackets after an orthodontic treatment. The method employed included a confocal laser microscope to capture the curved brackets' bases. The principal limitation of this study was that we had to exclude brackets with curvature higher than $85^{\circ}$. These are the first comparative data on the percentage of resin remnants on customized lingual brackets.

Lingual brackets showed a high frequency of ARI 2.

Even no differences between the four groups resulted statistically significant, we observed a slight tendency of more resin remnants on molar brackets, due to half-pad configuration. The debonding procedure requires significant attention to not damage the dental surface.

\section{Abbreviations}

ARI: Adhesive remnant index; SEM: scanning electron microscope

\section{Acknowledgements}

Not applicable.

\section{Authors' contributions}

GA coordinated the scanning procedures. CK and ADS performed the measurement. GB performed the data analysis. ADS, GB, MZ studied the methodology. AG and GAB coordinated the projects. All the authors equally contributed in writing the manuscript. All the authors read and approved the final manuscript.

Funding

There is no funding associated with this publication.

\section{Availability of data and materials}

The data used to support the findings of this study are available from the corresponding author upon request.

Ethics approval and consent to participate Not applicable.

Consent for publication

Not applicable.

\section{Competing interests}

There is no competing financial interest associated with this publication.

\section{Author details}

${ }^{1}$ Faculty of Dentistry, University of Padova, via Giustiniani 2 -, 35100 Padova, Italy. ${ }^{2}$ Department of Geoscience, University of Padova, Padova, Italy.

${ }^{3}$ Department of Orthodontics, University of Bologna, Bologna, Italy.

Received: 3 February 2020 Accepted: 30 April 2020

Published online: 14 May 2020

\section{References}

1. Schuler FS, van Waes H. SEM-evaluation of enamel surfaces after removal of fixed orthodontic appliances. Am J Dent. 2003;16(6):390-4.

2. Özer T, Başaran G, Kama JD. Surface roughness of the restored enamel after orthodontic treatment. Am J Orthod Dentofac Orthop. 2010;137(3):368-74.

3. Alessandri Bonetti G, Zanarini M, Incerti Parenti S, Lattuca M, Marchionni S, Gatto MR. Evaluation of enamel surfaces after bracket debonding: An invivo study with scanning electron microscopy. Am J Orthod Dentofac Orthop. 2011;140(5):696-702.

4. Hosein I, Sherriff M, Ireland AJ. Enamel loss during bonding, debonding, and cleanup with use of a self-etching primer. Am J Orthod Dentofacial Orthop. 2004;126(6):717-24.

5. Kitahara-Céia FMF, Mucha JN, dos Santos PAM. Assessment of enamel damage after removal of ceramic brackets. Am J Orthod Dentofac Orthop. 2008;134(4):548-55.

6. Wang WN, Meng CL, Tarng TH. Bond strength: A comparison between chemical coated and mechanical interlock bases of ceramic and metal brackets. Am J Orthod Dentofac Orthop. 1997;111(4):374-81. 
7. Sorel O, El Alam R, Chagneau F, Cathelineau G. Comparison of bond strength between simple foil mesh and laser-structured base retention brackets. Am J Orthod Dentofacial Orthop. 2002;122(3):260-6.

8. Chen C-S, Hsu M-L, Chang K-D, Kuang S-H, Chen P-T, Gung Y-W. Failure Analysis: Enamel Fracture after Debonding Orthodontic Brackets. Angle Orthod. 2008;78(6):1071-7.

9. Stratmann U, Schaarschmidt K, Wegener H, Ehmer U. The extent of enamel surface fractures. A quantitative comparison of thermally debonded ceramic and mechanically debonded metal brackets by energy dispersive microand image-analysis. Eur J Orthod. 1996;18(6):655-62.

10. Pont HB, Özcan M, Bagis B, Ren Y. Loss of surface enamel after bracket debonding: An in-vivo and ex-vivo evaluation. Am J Orthod Dentofac Orthop. 2010;138(4):387 e1-387.e9.

11. Bishara SE, Trulove TS. Comparisons of different debonding techniques for ceramic brackets: An in vitro study. Am J Orthod Dentofac Orthop. 1990; 98(3):263-73.

12. Ødegaard J, Segner D. Shear bond strength of metal brackets compared with a new ceramic bracket. Am J Orthod Dentofac Orthop. 1988;94(3):2016.

13. Habibi M, Nik TH, Hooshmand T. Comparison of debonding characteristics of metal and ceramic orthodontic brackets to enamel: an in-vitro study. Am J Orthod Dentofacial Orthop. 2007:132(5):675-9.

14. Joseph VP, Rossouw E. The shear bond strengths of stainless steel and ceramic brackets used with chemically and light-activated composite resins. Am J Orthod Dentofac Orthop. 1990;97(2):121-5.

15. Sha H-N, Choi S-H, Yu H-S, Hwang C-J, Cha J-Y, Kim K-M. Debonding force and shear bond strength of an array of CAD/CAM-based customized orthodontic brackets, placed by indirect bonding- An In Vitro study. PLoS One. 2018;13(9):e0202952.

16. Artun J, Bergland S. Clinical trials with crystal growth conditioning as an alternative to acid-etch enamel pretreatment. Am J Orthod. 1984;85(4):33340

17. Nwaneshiudu A, Kuschal C, Sakamoto FH, Rox Anderson R, Schwarzenberger K, Young RC. Introduction to Confocal Microscopy. I Invest Dermatol. 2012;132(12):1-5.

18. Bayguinov PO, Oakley DM, Shih C-C, Geanon DJ, Joens MS, Fitzpatrick JAJ. Modern Laser Scanning Confocal Microscopy. Curr Protoc Cytom. 2018; 85(1):e39.

19. Sfondrini MF, Gatti S, Scribante A. Shear bond strength of self-ligating brackets. Eur J Orthod. 2011;33(1):71-4.

20. Al Shamsi AH, Cunningham JL, Lamey PJ, Lynch E. Three-dimensional measurement of residual adhesive and enamel loss on teeth after debonding of orthodontic brackets: an in-vitro study. Am J Orthod Dentofacial Orthop. 2007:131(3):301 e9-15.

21. Arima VO, Vedovello Filho M, Valdrighi HC, Lucato AS, Santamaria M Jr, Vedovello SAS. Debonding forces of different pads in a lingual bracket system. Dental Press J Orthod. 2017;22(4):34-40.

22. Mavreas D, Cuzin J-F, Boonen G, Vande VB. The effect of various adhesives, enamel etching, and base treatment on the failure frequency of customized lingual brackets: a randomized clinical trial. Eur J Orthod. 2018:40(3):249-53.

23. Lombardo L, Kaplan A, Lapenta R, Bratti E, Pera C, Scuzzo G, Siciliani G. A comparative study of lingual bracket bond strength. Orthodontics (Chic). 2011;12(3):178-87.

24. Zanarini M, Gracco A, Lattuca M, Marchionni S, Gatto MR, Bonetti GA. Bracket base remnants after orthodontic debonding. Angle Orthod. 2013; 83(5):885-91.

\section{Publisher's Note}

Springer Nature remains neutral with regard to jurisdictional claims in published maps and institutional affiliations.

Ready to submit your research? Choose BMC and benefit from:

- fast, convenient online submission

- thorough peer review by experienced researchers in your field

- rapid publication on acceptance

- support for research data, including large and complex data types

- gold Open Access which fosters wider collaboration and increased citations

- maximum visibility for your research: over $100 \mathrm{M}$ website views per year

At $\mathrm{BMC}$, research is always in progress.

Learn more biomedcentral.com/submissions 